\title{
DETC2001/DAC-21069
}

\section{PERFECT STATIC BALANCE WITH NORMAL SPRINGS}

\author{
Freek L.S. te Riele \\ University of Twente \\ Department of Mechanical Engineering \\ Biomechanical Engineering Group, BMTI \\ P.O. Box 217, 7500 AE Enschede, The \\ Netherlands \\ email: f.l.s.teriele@wb.utwente.nl
}

\author{
Just L. Herder \\ Delft University of Technology \\ Department of Design and Engineering \\ HuMan-Machine Systems Group, MMS \\ Mekelweg 2, 2628 CD Delft, The Netherlands \\ email: j.I.herder@wbmt.tudelft.nl
}

\section{ABSTRACT}

In biomechanical engineering, gravity balancers are often used in orthoses carrying the weight of paralyzed limbs. In these applications, simplicity is an insuperable demand. However, known gravity balancers do not combine simplicity with perfect balance. This paper describes several gravity equilibrators providing perfect static balance. As opposed to many known solutions, the proposed balancers incorporate normal off-the-shelf springs, rather than the zero-free-length springs (springs with a length equal to zero when not preloaded or loaded externally) usually employed. The conceptional synthesis is presented, dimensional design criteria are derived, and prototypes are shown. Based on the prototype's working principle, an ankle prosthesis, which stabilizes the patient, will be developed.

Keywords: static balance, gravity equilibrator, rolling link mechanisms, rehabilitation technology, ankle prosthesis.

\section{INTRODUCTION}

In biomechanical engineering, gravity-balancing mechanisms are primarily used to compensate for the weight of weakened or paralyzed limbs. An example of a highly appreciated static balancing mechanism is the elbow orthosis shown in Figure 1 (Cool, 1976). The orthosis is intended to approximately balance the weight of the forearm. A slight underbalance causes the forearm to hang down when the upper arm is alongside the trunk. However, when the upper arm is moved, the spring action overcomes gravity, causing the forearm to lift up. A ratchet mechanism locks the forearm at 90 degrees and unlocks at reversed action. This makes it possible for the patient to control the lower arm by using the shoulder muscles.

The balancing mechanisms proposed in this paper also find their roots in the biomechanical engineering field. When a trans-tibial amputation occurs (amputation of part of the lower leg), a prosthetic ankle and foot have to replace the amputated part of the leg. Most ankle-foot prostheses are more or less rigidly connected to the lower leg. In order for a user to have more freedom of movement in standing and a more smoothly ambulation in walking, a flexible ankle joint is desired. In determining the rotational stiffness of such a flexible ankle joint, it was assumed that the motion of the Center of Body Mass during standing can be approximated by a rotation round the ankle and that the Center of Body Mass is statically balanced by the passive elastic structures around the ankle joint (Figure 2).

Many gravity balancing devices use zero-free-length springs (springs with a length equal to zero when not preloaded or loaded externally, e.g. Streit and Shin, 1993). However, considering that all anatomic structures which connect the lower leg to the foot have a non-zero free length, the aim was not to design a prosthetic ankle which statically balances the rotating Center of Body Mass using idealized springs. The desired ankle mechanism had to balance a mass rotating around a fixed point with normal springs (free length greater than zero). From this research an innovative prosthetic ankle joint concept has sprung which is able to perfectly statically balance a mass rotating around a fixed joint with the aid of technical springs (Riele et al., 2000). This paper is about the conception of the balancing mechanism of such a ankle prosthesis concept. 


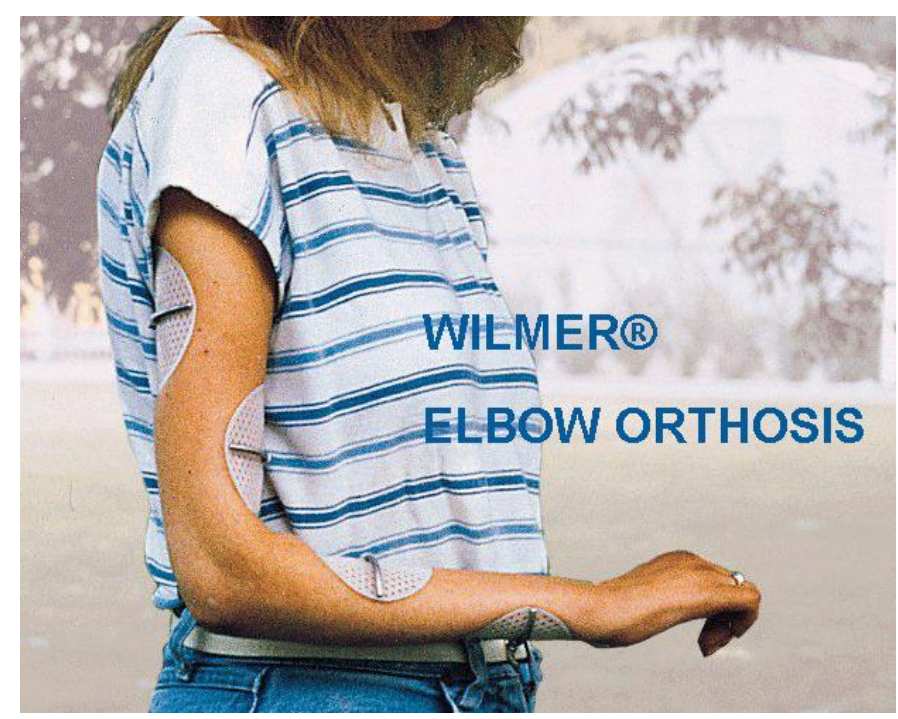

(a)

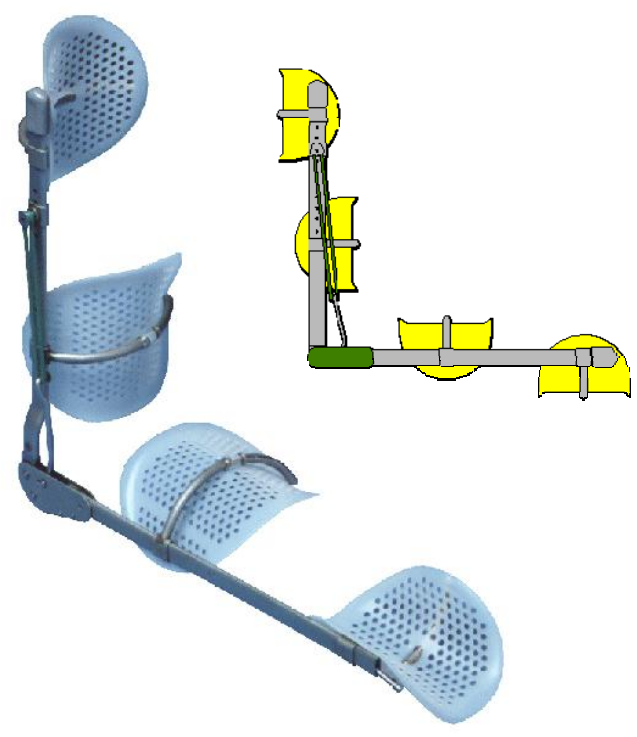

(c)

(b)

Figure 1. Wilmer elbow orthosis (Cool, 1976): (a) photograph of patient wearing the orthosis, (b) photograph of the orthosis, (c) diagram showing the rubber spring which counteracts the weigth of the forearm.

In gravity equilibrated mechanisms, several kinds of mechanisms can be distinguished. First there are balancers consisting of linkages with zero-free-length springs attached to the links, configured so as to provide perfect static balance. Other balancers contain mechanisms with normal springs (free length greater than zero), attached to linkages with stronger non-linear transfer functions, generally providing approximate static balance. Another group is formed by special mechanisms, such as wrapping cams, achieving perfect balance with normal springs. A brief review of these systems will be given.

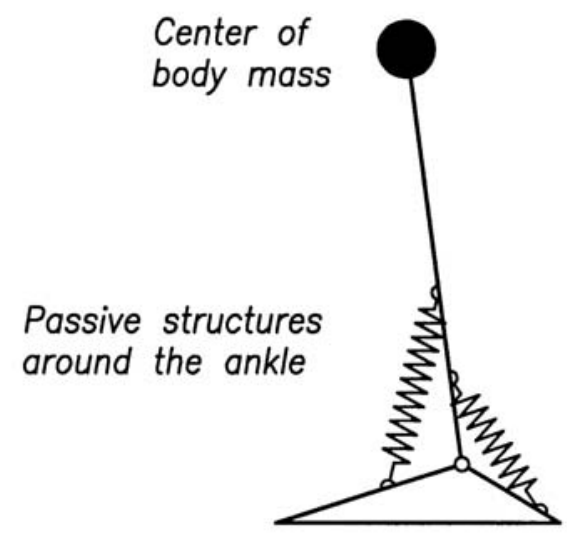

Figure 2. Schematic representation of prosthetic ankle-foot combination and the movement of the Center of Body Mass.

Many authors describe systems with mathematically perfect static balance incorporating zero-free-length springs. Hain (1952) used these springs to balance gravity forces acting on rotatable bodies, which was generalized for multiple springs by Haupt and Grewolls (1963). Hilpert (1968), Nathan (1985), Pracht et al. (1987), Streit and Shin (1993) expanded the theory to include additional links, for both open and closed loops, prismatic and revolute joints. Herder and Tuijthof (2000) presented a balanced arm, which can be used for a complete arm orthosis. Gosselin (1999), and Wang and Gosselin (1999, 2000) demonstrated the perfect static balancing of various parallel manipulators. In all of the above studies, zero-freelength springs in various embodiments were used to achieve perfect static balance against gravity, but also spring-to-spring balancers are known to be useful (Herder, 1998a). Normal springs generally result in approximate balance or require more complex adjustment mechanisms. Methodologies for optimizing static balance in linkages include those by Matthew and Tesar (1976), Mahalingam and Sharan (1986), Idlani et al. (1993), and Segla et al. (1998), while special adjustment mechanisms such as wrapping cams for balancing purposes are reported among others by Hain (1953), Ulrich and Kumar (1991), Fidweel (1992), and Hirose (1993).

This paper is about a distinct class of statically balanced systems. It will demonstrate that perfect static balance is also possible with normal springs, directly attached to the links of simple mechanisms. Prior to the derivation of the new systems, two spring-to-spring balanced systems with normal springs, invented by Carwardine (1932), will be revisited. Subsequently, this paper will develop one of them into a perfect solution for the equilibration of a mass. This will be pursued by using the approach and terminology set out in a previous paper (Herder, 1998b). First a solution with ideal 


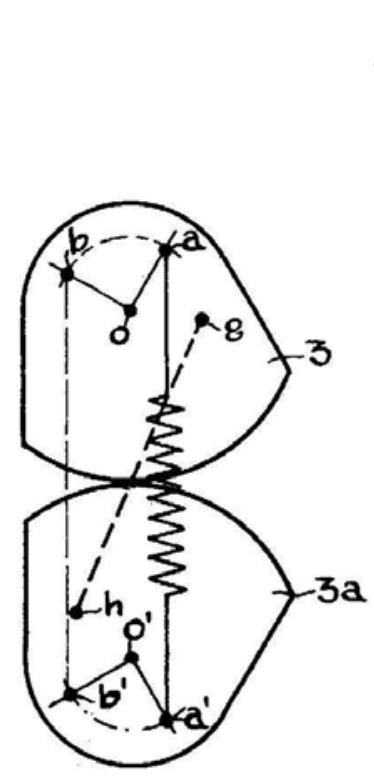

(a) (c)
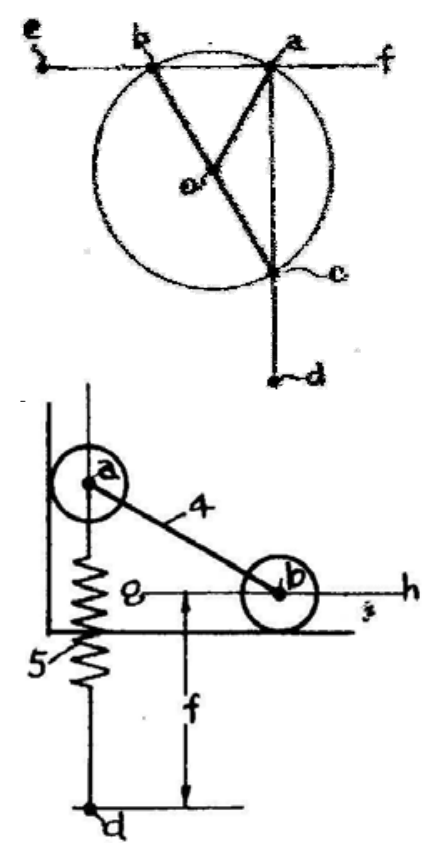

(b)
Figure 3. Original patent drawings of two perfect static spring to spring balancers incorporating normal springs (Carwardine, 1932), (a) rolling 8 figure, (b) sliding stepladder, (c) explanatory diagram, see text. Note: In (a) and (b) the contra springs are not drawn.

(zero free length) springs is given. Subsequently, the ideal springs are replaced by normal springs, the consequences of this action are investigated, and a practical version of a perfect balancer is given.

\section{NOMENCLATURE}

$\mathrm{a}=$ distance from pivot to spring attachment

$\mathrm{F}=$ Force

$\mathrm{k}=$ spring stiffness

$l_{0}=$ free length (length of spring when not preloaded or loaded externally)

$1_{1,2}=1_{0}+\mathbf{u}_{0}+$ strain of springs due to rotation of triangle

$\mathrm{m}=$ mass

$\mathrm{r}_{1,2}=$ distance from pivot to spring attachment

$\mathrm{r}=$ distance from fixed pivot to center of gravity of a mass

$\mathrm{R}=$ radius of rollers

$\mathrm{u}_{0}=$ pre-strain length of a spring

$\mathrm{V}=$ potential energy

$\alpha=$ angle between lines from pivot to spring attachment points

$\beta=$ angle between lines from pivot to spring attachment points

$\varphi=$ rotation angle

$\psi=$ angle between symmetry line and line from pivot to spring attachment point



(a)

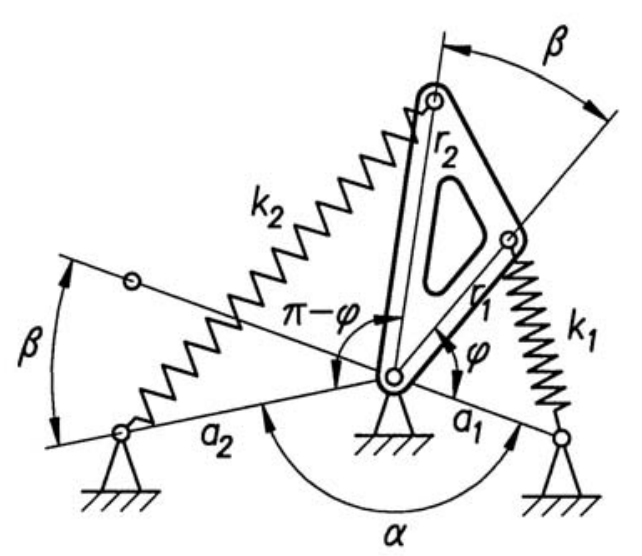

(b)

Figure 4. Statically balanced spring to spring mechanisms: (a) basic configuration, (b) modified according to the rotation rule (Herder, 1998), where element indexed 2 is rotated by the angle $\beta$.

\section{BACKGROUND}

Two mechanisms in a patent by Carwardine (1932) appear to be the first if not the only ones reported in literature with perfect static balance and normal springs (Figure 3ab). Evidently, geometric insight triggered the invention: "It follows from the 31st proposition in the third book of Euclid that since the path of point $a$ is a circle then angle bac is always a right angle and angle bao is always the trigonometric complement of angle oac" (figure 3c). Carwardine concludes with the statement that the case where "the said points on a said movable member are constrained to move instantaneously in directions which are always mutually at right angles to one another", is a condition for the static balance of this kind of systems.

Independently, and ignorant of Carwardine's patent, the same working principle in a similar embodiment was found by employing a different approach. In a previous paper (Herder, 1998b) it was argued that many different statically balanced systems can be conceived in a lucid and logical way by applying modification rules to an elementary basic configuration, based on the notion that constant potential energy implies static balance. The basic configuration consists 


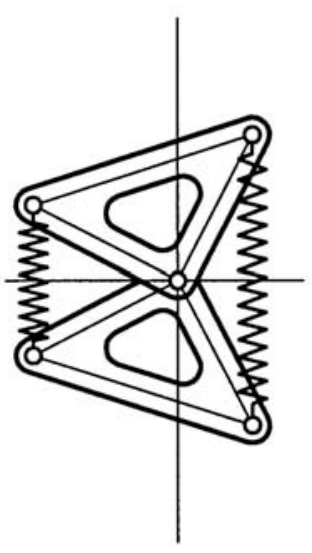

(a)

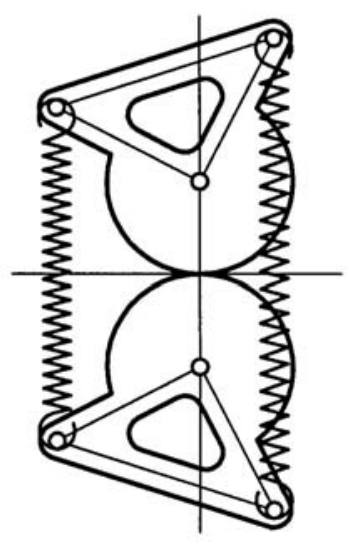

(c)

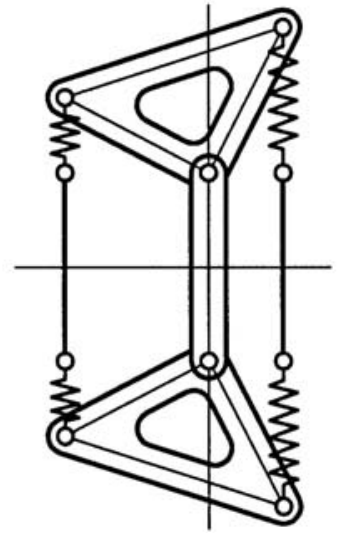

(b)

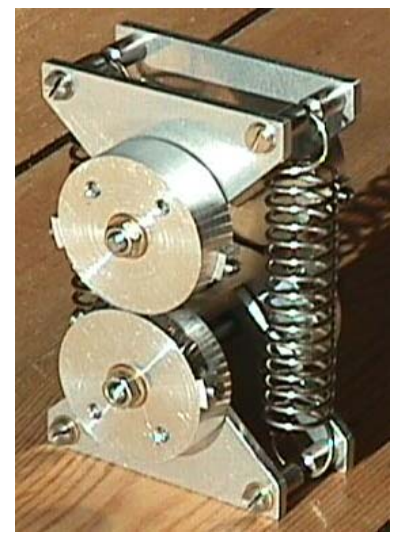

(d)
Figure 5. Conception of a spring-to-spring balancer with normal springs and yet perfect balance: (a) basic configuration, modified through rotation of the spring-lever-elements by 90 degrees relative to one another, shown in a deflected position, (b) the triangles shifted apart by a distance $d$, and ideal springs replaced by normal springs with $l_{0}=d$, (c) rolling version with $l_{0}=d=2 R$, (d) photograph of a working model of the spring force balancer, showing the glossy bands wrapped around the rollers.

of a rotatable lever and two ideal springs $\left(\ell_{0}=0\right)$ according to Figure 4a. The total potential energy $V$ in the springs is given by:

$$
V=V_{1}+V_{2}=\frac{1}{2} k_{1} \ell_{1}^{2}+\frac{1}{2} k_{2} \ell_{2}^{2}
$$

where $\ell_{1}$ is the length of spring $k_{1}$, and $\ell_{2}$ is the length of spring $k_{2}$. Using the cosine rule, and rearranging gives:

$$
\begin{aligned}
& V=\frac{1}{2} k_{1}\left(a_{1}^{2}+r_{1}^{2}\right)-k_{1} a_{1} r_{1} \cos \varphi+\frac{1}{2} k_{2}\left(a_{2}^{2}+r_{2}^{2}\right) \\
& -k_{2} a_{2} r_{2} \cos (\pi-\varphi)
\end{aligned}
$$

Evidently, the potential energy is constant under the condition:

$$
k_{1} a_{1} r_{1}=k_{2} a_{2} r_{2}
$$

Using Equation 3, it is readily seen that the case of Figure 4a is statically balanced when $k_{1}=k_{2}$ and $a_{1}=a_{2}=r_{1}=r_{2}$. Therefore every position is an equilibrium position.

Regarding the mechanism in Figure $4 \mathrm{a}$ as a combination of two spring-lever-elements, one indexed 1 and the other indexed 2, allows for creative manipulation, which can lead to many different statically balanced systems by using modification rules (Herder, 1998b). An example is given in Figure $4 \mathrm{~b}$, where the spring-lever-elements are rotated apart relative to one another by an angle $\beta$. As this has no consequences for the potential energy functions of the springs, perfect static balance is maintained. Inspection of the figure leads to the geometric condition:

$$
\alpha+\beta=\pi
$$

This condition must be satisfied in addition to Equation 3, also in the more general case when $k_{1} \neq k_{2}$ and $a_{1} \neq a_{2}$, and $r_{1} \neq r_{2}$ (see also Hoek, 1986).

Figure 5a shows another modified form of the basic configuration. The left and right halves are rotated apart by 90 degrees $(\alpha=\beta=\pi / 2)$. Since, in this case, the lengths of the levers and the distance from the pivot to the fixed spring ends all amount to $a$, two equal isosceles triangles result. The figure shows the mechanism in a deflected position, drawn symmetrically about the horizontal line through the pivot. The next step (Figure 5b) is to distend the two triangles by a distance $\mathrm{d}$, and replace the ideal springs with springs having a free length $l_{0}=d$. When the added link is fixed, clockwise rotation of one of the triangles by an angle $\varphi$ has to induce a counterclockwise rotation of the other triangle by the same amount. Therefore, the triangles rotate by an angle of $2 \varphi$ with respect to one another. A profitable practical embodiment is to replace the distention link by two circular rollers of radius $R$, such that $2 R=d$ (Figure $5 \mathrm{c}$ ). Non-slipping rollers or gears guarantee correct motion, while the two members are being pressed together by the springs. A working model was made as shown in Figure 5d, where flexible bands are wrapped between the rollers to prevent the rollers from slipping. Noteworthy is the fact that motion of the real system is restricted to $-45<\varphi<45$ degrees, due to the fact that the helical tension springs used cannot effectively push when shorter than $\ell_{0}$.

\section{GRAVITY BALANCER}

The previous section demonstrated that perfect spring-to-spring balance was achieved with normal springs when the triangles in Figure $5 \mathrm{~b}$ are distended by a distance equal to the free length $l_{0}$ of the springs. When normal springs of stiffness $k$ and free length $l_{0}$ are introduced into the configuration of Figure $5 \mathrm{a}$, or 


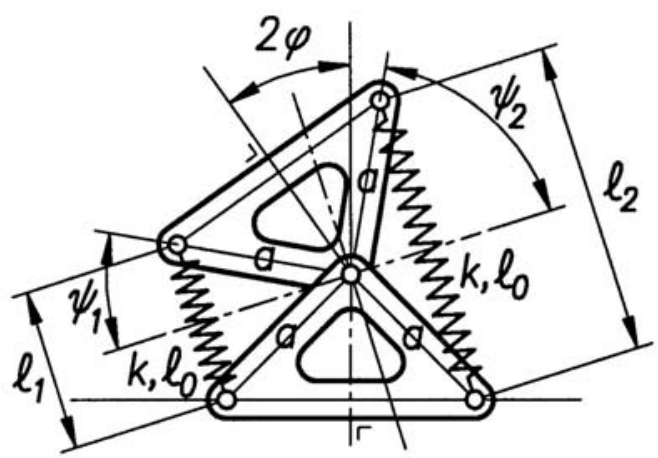

Figure 6. Configuration of figure 5a, now with normal springs, and deflected by an angle of $2 \varphi$.

when the condition of $l_{0}=d$ is not satisfied in the configuration of Figure $5 \mathrm{~b}$, a residual moment will be present which is a function of the angle $\varphi$, as will be demonstrated next.

Using the symbols shown in Figure 6, where it is especially noted that the upper half of the mechanism is rotated by an angle of $2 \varphi$, the following geometrical relations can be identified:

$$
\psi_{1}=\frac{\pi}{4}-\varphi ; \quad \psi_{2}=\frac{\pi}{4}+\varphi ; \quad \ell_{1}=2 a \sin \psi_{1} ; \quad \ell_{2}=2 a \sin \psi_{2}
$$

while the cosine rule reveals:

$$
\ell_{1}^{2}=2 a^{2}(1-\sin 2 \varphi) ; \quad \ell_{2}^{2}=2 a^{2}(1+\sin 2 \varphi)
$$

The total potential energy, considering that equal springs of stiffness $k$ and free length $l_{0}$ are used, yields:

$$
V=\frac{1}{2} k\left(\ell_{1}^{2}+\ell_{2}^{2}-2 \ell_{0}\left(\ell_{1}+\ell_{2}\right)+2 \ell_{0}^{2}\right)
$$

Using the geometric equality:

$$
\sin \left(\frac{\pi}{4}-\varphi\right)+\sin \left(\frac{\pi}{4}+\varphi\right)=2 \sin \frac{\pi}{4} \cos \varphi=\sqrt{2} \cos \varphi
$$

and substituting Equations 5 and 6 into Equation 7, gives:

$$
V=k\left(2 a^{2}+\ell_{0}^{2}\right)-2 \sqrt{2} k a \ell_{0} \cos \varphi
$$

The pre-strain length $\mathrm{u}_{0}$ of the springs (in the symmetric position of the triangles) can be derived from the zero length $1_{0}$ of the springs:

$$
u_{0}=\sqrt{2} a-l_{0} \Rightarrow l_{0}=\sqrt{2} a-u_{0}
$$

Substituting Equation (10) in Equation (9) gives for the potential energy V:

$$
V=k\left(2 a^{2}+\left(\sqrt{2} a-u_{0}\right)^{2}\right)-2 \sqrt{2} k a\left(\sqrt{2} a-u_{0}\right) \cos \varphi
$$

Examining Equations 9 and 11, it is observed that the potential energy is constant only if the cosine-term vanishes. Consequently, static balance is achieved only if $l_{0}=0$ or

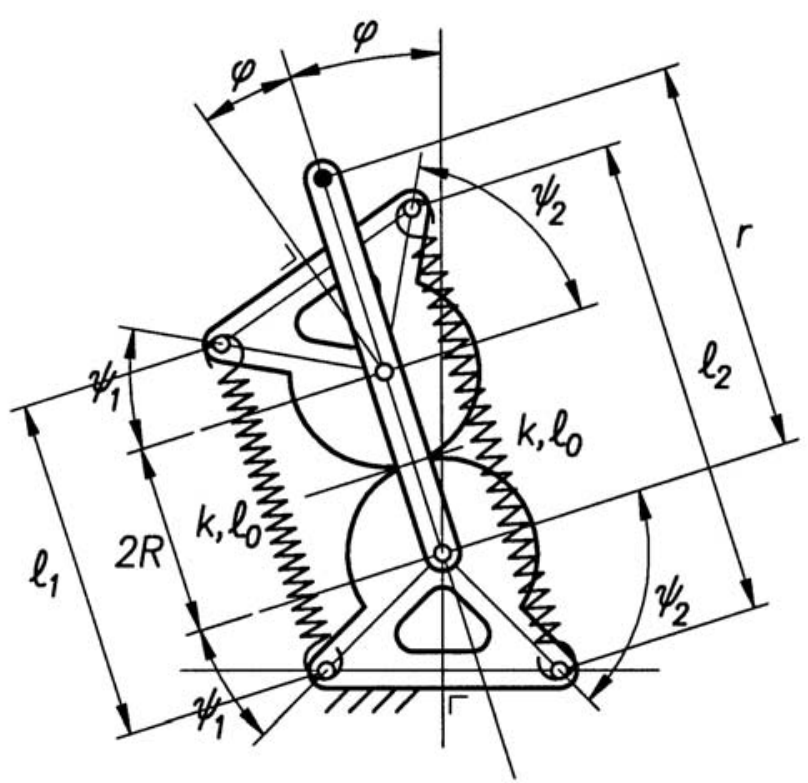

(a)



(b)

Figure 7. (a) configuration according to Figure 5c, with extended link to carry a mass, (b) as (a) with triangles overlapped in stead of distended.

$u_{0}=\sqrt{2} a$, otherwise a moment remains. This moment is found as the derivative of the potential energy with respect to the rotation of the upper triangle $2 \varphi$, according to:

$$
M=\frac{\partial V}{\partial 2 \varphi}=\frac{1}{2} \frac{\partial V}{\partial \varphi}=\sqrt{2} k a l_{0} \sin \varphi=\sqrt{2} k a\left(\sqrt{2} a-u_{0}\right) \sin \varphi
$$

Since a mass, rotating about a fixed point, exerts a sinusoidal moment, the remaining moment of Equation 12 can be used to statically balance a mass, provided a mechanism is conceived which rotates the mass about a fixed point within the mechanism according to an angle one half of the angle rotation between the triangles. Inspection of Figure $5 \mathrm{~d}$ reveals that the 


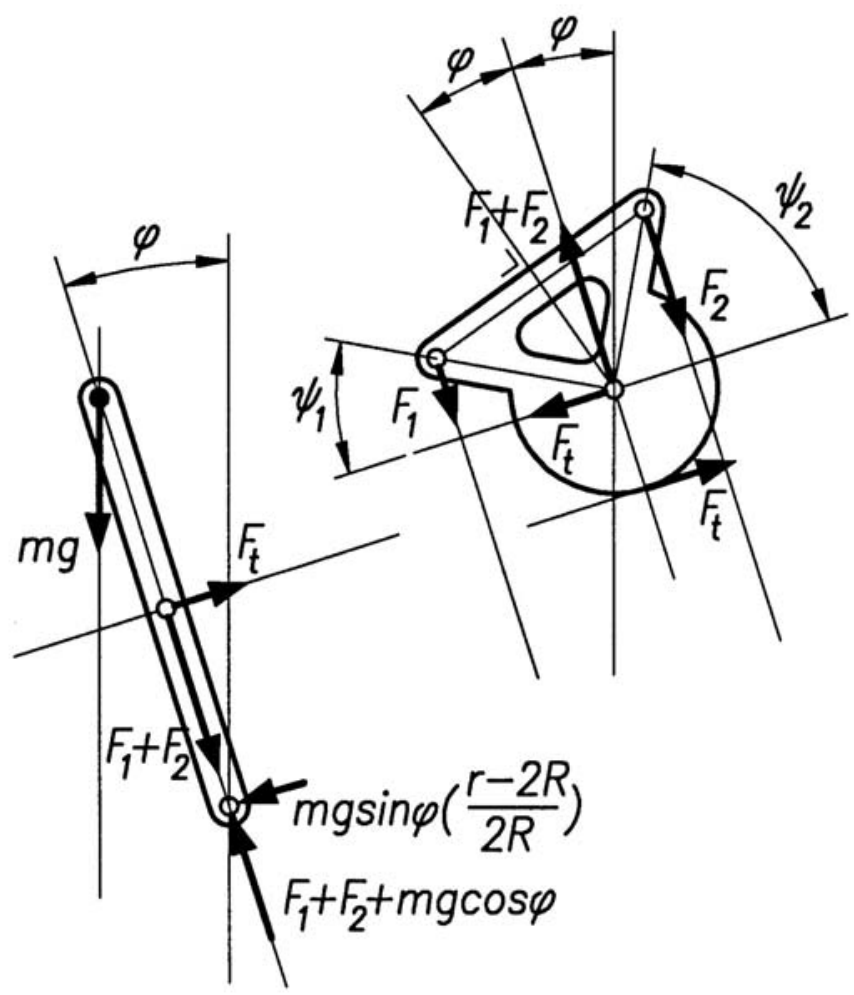

(a)

(b)

Figure 8. Force analysis of the mechanism in Figure 7a, (a) link, (b) upper roller.

link in between the triangles performs just so, provided rollers (or gears) are present to ensure the single degree-of-freedom of the mechanism. This is illustrated in Figure $7 \mathrm{a}$ and $7 \mathrm{~b}$. The triangles have been shifted apart/ through each other by a distance 2R, and the link holding them apart/ together is extended to carry a mass $\mathrm{m}$, such that the mass is located at a distance $r$ from the fixed pivot.

Assuming a pre-strain length $\mathrm{u}_{0}$ of the springs, the potential energy of the springs in the mechanisms drawn in Figure 7 can be described by Equation 11. The total potential energy of the mechanisms is the sum of the potential energy of the springs and of the rotating mass. Thus:

$$
\begin{aligned}
& V_{\text {total }}=V_{\text {springs }}+V_{\text {mass }}=k\left(2 a^{2}+\left(\sqrt{2} a-u_{0}\right)^{2}\right) \\
& -2 \sqrt{2} k a\left(\sqrt{2} a-u_{0}\right) \cos \varphi+m g r \cos \varphi
\end{aligned}
$$

This expression becomes constant for every rotation $\varphi$ when the cosine terms vanish, thus:

$$
-2 \sqrt{2} k a\left(\sqrt{2} a-u_{0}\right)+m g r=0
$$

This condition shows that the appropriate combination of mass, spring parameters, and geometry yield perfect static balance for infinite cases. In fact, any two equal linear springs may be

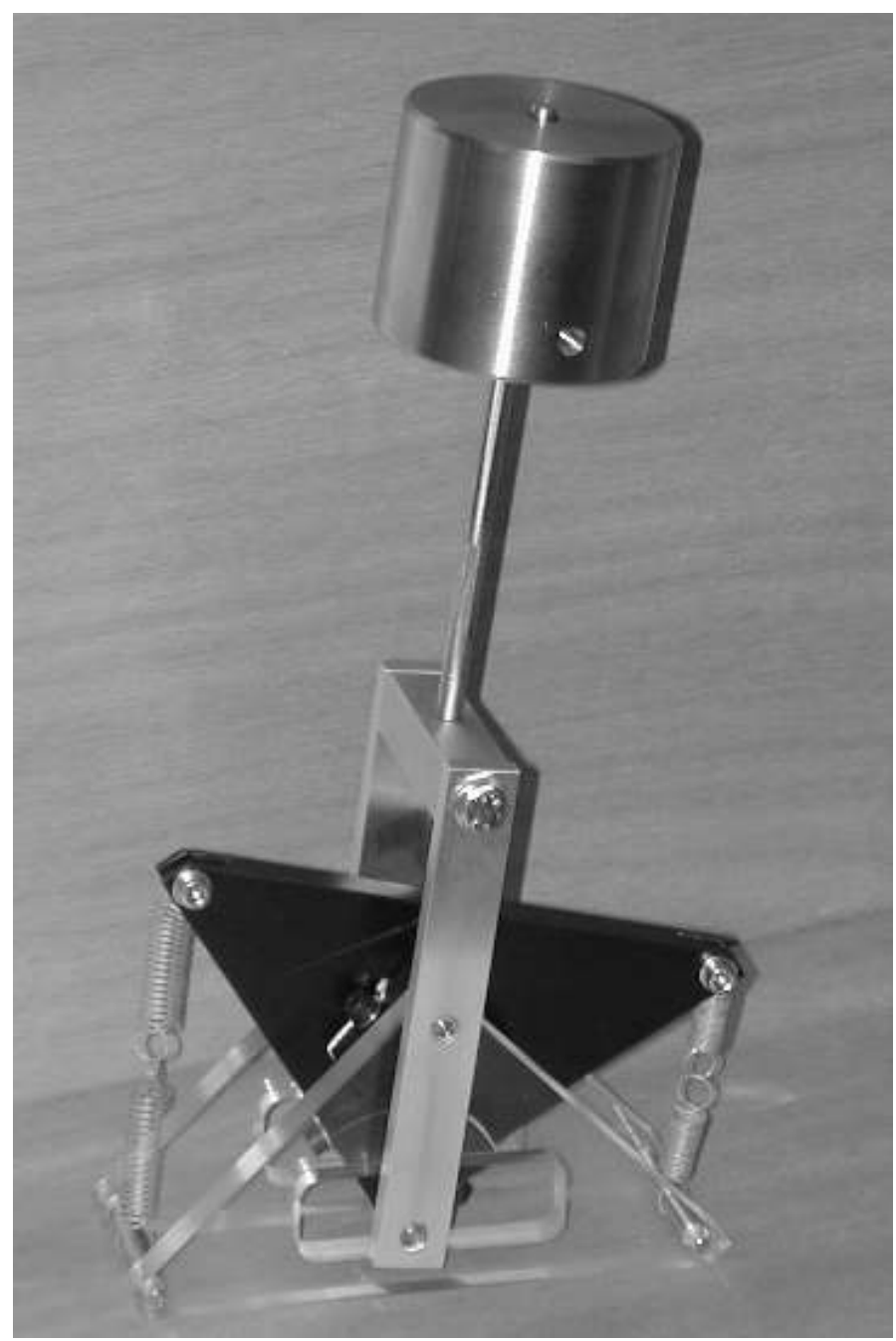

Figure 9. Photograph of the working model of the gravity equilibrator.

used since adjusting $r$ can always restore perfect balance due to the linear nature of the expression. Again it is seen that when $u_{0}$ equals $\sqrt{2} a$ or $2 R$ equals $l_{0}$, the only solution for static balance is the trivial case of $r=0$.

A force analysis was carried out in order to verify the energy approach in the conception phase. Figure 8 gives the free-body diagrams of the link and the upper triangle. It is convenient to resolve the forces in components parallel to the link and components perpendicular to the link. From the moment equilibrium of the link about the fixed pivot (bottom of the link), it is seen that a component $F_{t}$ in the upper pivot is required perpendicular to the link (Figure 8a):

$$
F_{t}=m g \frac{r \sin \varphi}{2 R}
$$

The reaction-force acts at the center of the upper roller. For equilibrium, an equal but opposite force is required in the 
contact with the other roller. Furthermore, it is assumed that the forces parallel to the link $\left(F_{1}+F_{2}\right)$ are supported by the pivot. The moment equilibrium about the contact point then yields (Figures $8 \mathrm{~b}$ and $8 \mathrm{c}$ ):

$$
F_{1} a \cos \psi_{1}-F_{2} a \cos \psi_{2}+F_{t} R=0 \Rightarrow-M_{\text {springs }}+F_{t} R=0
$$

Substituting Equation 12 as the moment round the contact point caused by the springs and solving for $F_{t}$ gives:

$$
F_{t}=\sqrt{2} \frac{k a}{R}\left(\sqrt{2} a-u_{0}\right) \sin \varphi
$$

Combining Equations 15 and 17 results in the same balancing condition as Equation 14. The force analysis reveals that a considerable tangential force $F_{t}$ is present between the rollers. A similar analysis can be carried out for the mechanism given in Figure $7 b$.

Figure 9 shows a working model of the gravity balancer concept, proposed for use as a prosthetic ankle joint by Riele et al (also see Figure 7b). Note that in this version the rollers are not pressed together but pushed away from one another. The link, which also carries the mass, contains bearings for the rollers and takes up the forces parallel to the link. Flexible cables in-between the rollers prevent the rollers from slipping. This model functions quite satisfactory. Provided it is placed on a horizontal base, the link is in equilibrium throughout its range of motion, while friction is negligible.

\section{CONCLUSION}

The use of normal springs in static balancing generally result in approximate balance or require more complex adjustment mechanisms. However, when static gravity balance is pursued in biomechanics, simplicity of a mechanism is an insuperable demand.

This paper discussed a special class of statically balanced mechanisms, which provide perfect balance with normal springs. It was demonstrated that any mass, tracing a circle about a fixed pivot, could be perfectly statically balanced using two equal normal (non-zero-free-length) springs in a special configuration. Advantages are that off-the-shelf springs can be used so that the special constructions (and the weight and friction associated with these) or special coiling techniques required to obtain zero-free-length behavior, or special mechanisms such as wrapping cams, can be avoided. However, as opposed to the perfect equilibrators with ideal (zero-freelength) springs, the solutions in this class cannot turn a full 360 degrees.

The gravity balancing concept presented has a relatively simple working principle and can be manufactured compactly (Figure $7 \mathrm{~b}$ and 9). Therefore the concept seems suitable for further development into an ankle prosthesis which stabilizes the patient, fully or partly, according to its gravity compensating effect. However, the fact that the proposed concept is only able to balance a mass $2 \mathrm{D}$ must be seen as a disadvantage, as an ankle is distinctly a 3D joint. Finally it should also be noted that although a relative simple working principle has been developed for achieving perfect static gravity balancing with normal springs, the question rises if the Center of Body Mass has to be perfectly balanced as approximate gravity balancing results in even more simple and compact to manufacture working principles.

\section{ACKNOWLEDGMENTS}

The author would like to thank the Dutch Science Foundation S.T.W. for funding the ankle-foot prosthesis research done at the Bio-Mechanical Engineering group of the University of Twente from which this gravity balancing concept is a direct result. Thanks to E.E.G. Hekman, T.G.M. Krone and J.C. Cool of the University of Twente for their part in the research. The first author would especially like to thank the co-author Just Herder for the initiative to write a paper about this gravity balancing concept and for the large amount of work he has put in writing this paper. Finally the authors would like to compliment the reviewers on their work.

\section{REFERENCES}

Carwardine G (1932) Improvements in elastic force mechanisms, UK Patent 377.251, Specifications of Inventions, Vol. 2773, Patent Office Sale Branch, London.

Cool JC (1976) An elbow orthosis, Biomedical Engineering, 11(10)344-347.

Fidweel PH, Bandukwala N, Dhande SG, Rheinholz CF, Webb G (1992) Synthesis of wrapping cams, Proceedings of 22nd Biennial Mechanisms Conference ASME, Robotics, Spatial Mechanisms and Mechanical Systems, ASME Design Engineering Division DE v 45 p337/343.

Gosselin CM (1999) Static balancing of spherical 3-dof parallel mechanisms and manipulators, The International Journal of Robotics Research, 18(8)819-829.

Hain K (1952) Der Federausgleich von Lasten, Grundlagen der Landtechnik, (3)38/50.

Hain K (1953) Gelenkarme Bandgetriebe für den Kraftausbleich durch Federn, Grundlagen der Landtechnik, (4)100/9.

Haupt G, Grewolls J (1963) Über das Gleichgewicht zwischen Federkräften und konstanten Kräften, Maschinenbautechnik 8(12)423/8.

Herder JL (1998a) Design of spring force compensation systems, Mechanism and Machine Theory, 33(1-2)151-161.

Herder JL (1998b) Conception of balanced spring mechanisms, CDROM proceedings ASME DETC 25th Biennial Mechanisms Conference, Sept 13-16, Atlanta, Georgia, paper number DETC98/MECH-5934.

Hilpert H (1968) Weight balancing of precision mechanical instruments, Jnl Mechanisms, (3)289/302. 
Hirose S (1993) Biologically inpired robots, Oxford University Press.

Hoek, W van der (1986) A mechanical designer's case book: DDP, Philips CFT Report 59/85, Compiled by the Mechanical Engineering Dept of the Eindhoven University of Technology.

Idlani S, Streit DA, Gilmore BJ (1993) Elastic Potential Synthesis, A generalized procedure for dynamic synthesis of machine and mechanism systems, Journal of Mechanical Design, 115(3)568/75.

Mahalingam S, Sharan AM (1986) The optimal balancing of the robotic manipulators, IEEE international conference on robotics and automation, p828-835

Matthew GK, Tesar D (1976) Synthesis of spring parameters to balance general forcing functions in planar mechanisms, ASME-paper 76-DET-45.

Nathan RH (1985) A constant force generating mechanism, Journal of Mechanisms, Transmissions, and Automation in Design, 107(12)508/12.

Pracht P, Minotti P, Dahan M (1987) Synthesis and balancing of cam-modulated linkages, ASME Design and Automation Conference, 10(2)221/6.

Riele FLS te, Hekman EEG, Cool JC (2000) The development of a highly efficient energetic locomotion foot, In: Bante I,
Feijen J (eds) Proceedings of the Dutch Annual Conference on BioMedical Engineering, Oct 9-10, Papendal, The Netherlands, p112-113.

Segla S, Kalker-Kalkman CM, Schwab AL (1998) Statical balancing of a robot mechnaism with the aid of a genetic algorithm, Mechanism and Machine Theory, 3(1/2)163174.

Streit DA, Shin E (1993) Equilibrators for planar linkages, Journal of Mechanical Design, 115(3)604/11.

Tuijthof GJM, Herder JL (2000) Design, actuation and control of an anthropomorphic robot arm, Mechanism and Machine Theory 35(7)945-962.

Ulrich N, Kumar V (1991) Passive mechanical gravity compensation for robot manipulators, Proceedings of the 1991 IEEE International Conference on Robotics and Automation, Sacramento, California, April 1991, p15361541.

Wang J, Gosselin CM (1999) Static balancing of spatial threedegree-of-freedom parallel mechanisms, Mechanism and Machine Theory, Vol. 34, p437-452.

Wang J, Gosselin CM (2000) Static balancing of spatial fourdegree-of-freedom parallel mechanisms, Mechanism and Machine Theory, Vol. 35, p563-592. 